\title{
Smart Solar-Powered Lighting System for Smart Cities
}

\author{
Sairoop Dasaraju, Hamid Shahnasser* \\ College of Science and Engineering, San Francisco State University, San Francisco, USA. \\ * Corresponding author. Tel.: 415-338-2124; email: hamid@sfsu.edu \\ Manuscript submitted November 10, 2019; February 2, 2020. \\ doi: 10.17706/ijcce.2020.12.2.83-92
}

\begin{abstract}
Over 300 million streetlights in cities and campuses throughout the world are powered using electricity generated by burning fossil fuels. Traditional streetlights have mechanical timers to turn the lights ON-OFF for a preset amount of time. Since they are manually operated, they are turned on at full intensity for a fixed period of time regardless of the amount of ambient light, leading to energy wastage. Another disadvantage of these networks is that there is no way of detecting a light when it stops functioning and it takes a lot of time to identify, service and replace it. This paper discusses an off-grid solar powered lighting system for smart cities that is economic, and efficient. The designed system powers DC LED lights. LEDs are used since their power consumption is much less compared to the enormous power consumption of traditional HID lights. The system is integrated with the IoT enabling it to take intelligent decisions without any human involvement. Further, the use of IoT enables remote control and monitoring of the system using Android and iOS applications. The system utilizes sensors to obtain data on the amount of power generated by the solar panels and the amount of power consumed by the LEDs. This data can be monitored using the mobile applications and it is also backed up in a database on the Google Cloud. This data can be analyzed to obtain insights on power generation and power consumption trends. The proposed system is implemented economically, and the performance analysis results are promising compared to traditional lighting systems.
\end{abstract}

Key words: Smart lighting system, smart cities, solar power, Wi-Fi, Android and iOS applications.

\section{Introduction}

As of 2017, globally 70\% of all electricity was generated by burning fossil fuels [1], a source of air pollution and greenhouse gases. Globally there are approximately 300 million streetlights using this electricity generated from burning fossil fuels [2]. The use of renewable resources to power streetlights has only just begun. According to a survey done by Navigant Research there are 6 million renewable energy powered streetlights around the world and the number is expected to go up to 73 million by 2026 . Although it is impossible to use solar power in every part of the world it is one of the most widely used and abundant source of renewable energy.

A major part of energy supplied to cities around the world is consumed by streetlights and path lights. According to the statistics discussed above, most of the lights in cities around the world are still manually operated. These lighting systems use HID lamps or high-pressure sodium lamps as the light source which consume an enormous amount of energy and release a lot of heat. Since they are manually operated many problems like maintenance issues, timing and connectivity issues arise. These problems are being addressed using technologies like LEDs and IoT (Internet of Things) etc. Majority of streetlighting control 
systems are still running in a manual setup. The use of IoT enables automation of any system and helps simplify various problems involved in an otherwise manual setups.

Errors are inevitable in manual setups which in turn lead to energy wastage and reduced performance [3]. The aim of this paper is to build and test the design of a streetlighting system that uses solar power and IoT to increase productivity and efficiency in a cost-effective manner while enabling wireless control and monitoring. The system is off grid and uses only solar panels for powering up the lights. This ensures that there is no energy wastage, reduces the $\mathrm{CO} 2$ emissions and reduces the damage to the environment. LEDs are chosen as the light source because of their various advantages over the traditional HID lamps like power saving, reduced maintenance and durability [4], [5].

To replace the traditional mechanical timer, a daylight sensor is used. The day light sensor ensures that light only turns on after sunset and turns off after sun rise reducing the energy wastage. Further, current measuring sensors are used to calculate the power generated by the panels and the power consumed by the load. This data is stored in a database on the cloud over the internet. An Android/iOS mobile application is developed and integrated with the system for wireless control and monitoring. The lighting system design proposed allows greater monitorability, control and good performance.

\section{Existing Technologies and Challenges}

There are many existing products that harness solar energy to power street lighting networks. Some of the prominent companies in this market today are Soluxio, EnGoPlanet and Victron Energy. Soluxio uses a patented cylindrical solar panel design [6] to power their streetlights shown in Fig. 1(a). They provide remote control and remote monitoring features using Zigbee and GPRS. While EnGoPlanet uses unique, semi-flexible solar panels designed by Victron energy to power their lights shown in Fig. 1(b). These lights use Wi-Fi for remote control and monitoring and have additional sensors to collect and relay temperature and air pollution data. All these products have solar powered lighting systems that have a stand-alone solar panel and the design is implemented within each individual light. Allocating resources to every single light is an inefficient way of using them and makes these systems very expensive to be implemented on a large scale. The proposed lighting system design is of a control hub that can be used to power LEDs. This system is integrated with an Android/iOS cross platform mobile application for remote control and surveillance. The design is made so that the resources are shared among multiple LEDs and they can all be controlled and monitored from a single mobile application. The mobile application addresses the accessibility issue by sending notifications about critical events like system malfunction or LED burnout in real time. Further, the modular design of the system makes the process of adding additional sensors or peripherals effortless.

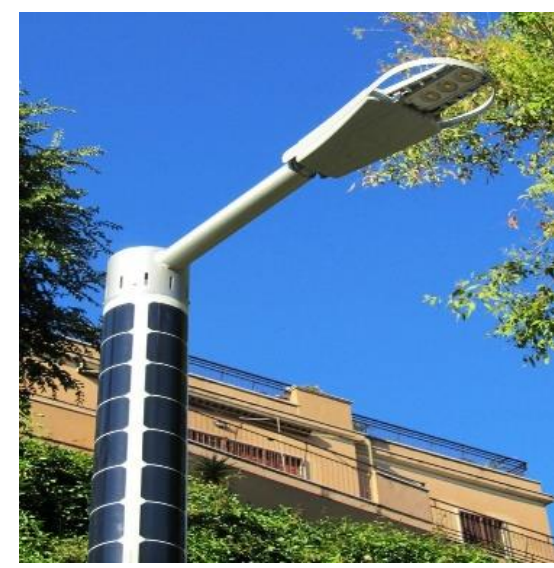

(a)

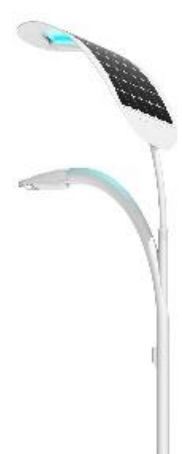

(b)

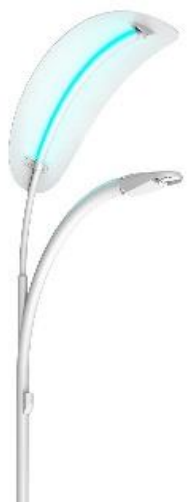

Fig. 1. (a) Soluxio's cylindrical solar light; (b) EnGoPlanet's streetlight with curved solar panel. 


\section{Architecture and the Cost of Making the System}

The components used to implement the proposed design are listed in Table 1. The proposed system has a simple and modular design to facilitate easy maintenance, scalability and faster integration of additional sensors and peripherals. The following sections discuss each module of the design in detail.

Table 1. List of Components and Their Prices

\begin{tabular}{|l|l|l|}
\hline S.no & Name of the component & Price \\
\hline 1. & 50W Solar panel & $\$ 110$ \\
\hline 2. & Solar panel mount & $\$ 174$ \\
\hline 3. & Solar charge controller & $\$ 40$ \\
\hline 4. & 18650 battery & $\$ 200$ \\
\hline 5. & Hardware enclosure & $\$ 60$ \\
\hline 6. & LED light & $\$ 30$ \\
\hline 7. & NodeMCU & $\$ 10$ \\
\hline 8. & Battery management system & $\$ 14$ \\
\hline 9. & Daylight sensor & $\$ 7$ \\
\hline 10. & Electronic components & $\$ 25$ \\
\hline 11. & Connectors & $\$ 25$ \\
\hline 12. & Miscellaneous & $\$ 15$ \\
\hline & TOTAL & $\$ 710$ \\
\hline
\end{tabular}

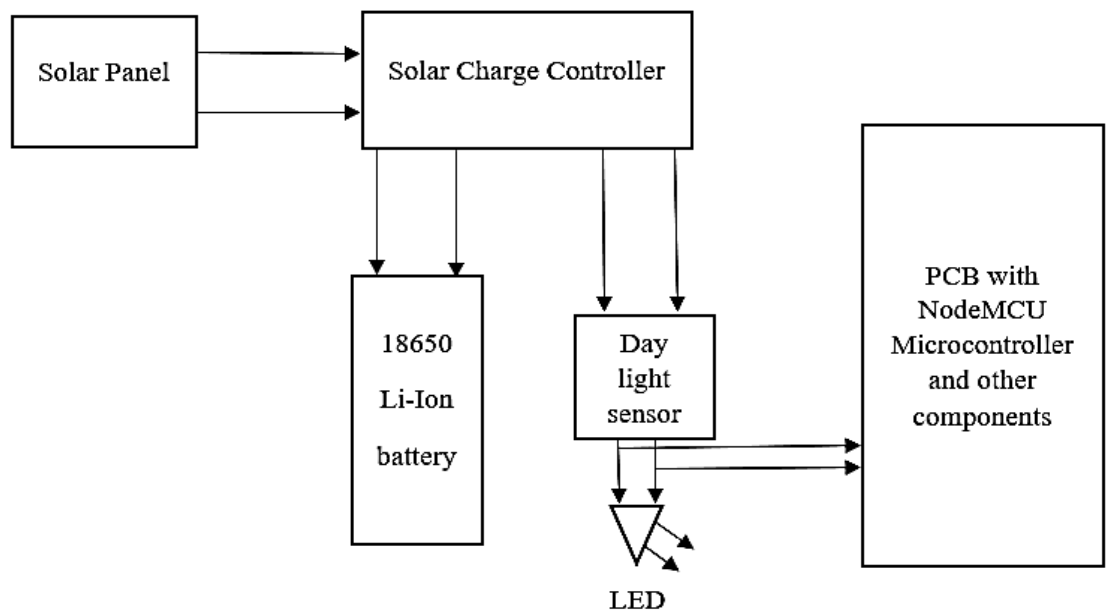

Fig. 2. Architecture of the system.
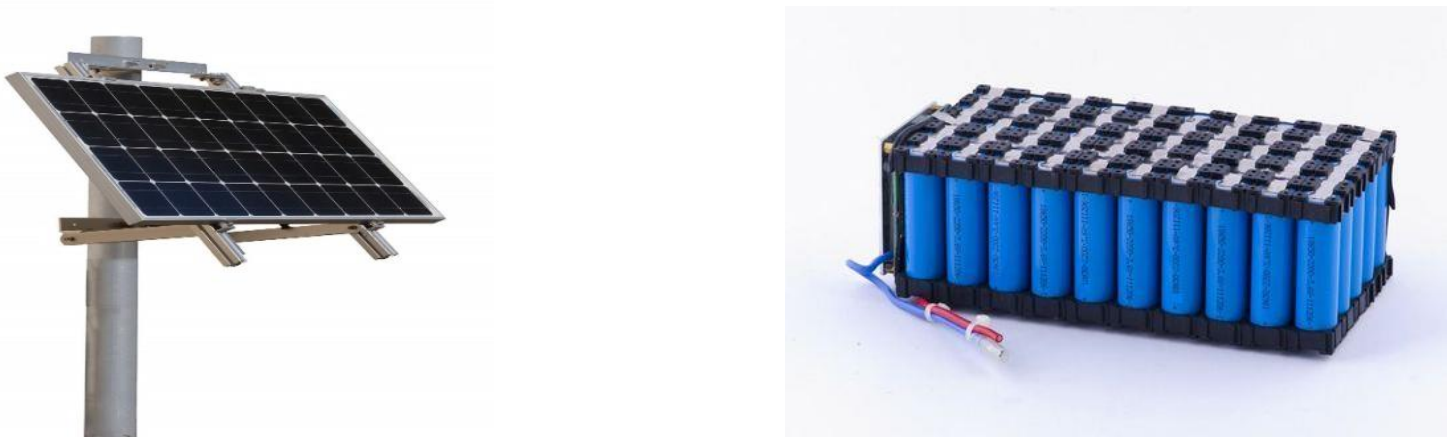

Fig. 3. A solar panel mounted on the side of a pole. Fig. 4. Li-ion battery pack made using 18650 cells. 
This paper presents an economic and efficient design of a solar powered streetlighting system. Fig. 2 shows the architecture of the proposed system. This design consists of a control hub with 4 modules: a solar charge controller that acts a bridge between the solar panel (Fig. 3), the battery shown in Fig. 4, the load, and the PCB that enables wireless control.

\section{Lighting System Modules}

The smart solar lighting system is divided into four modules. In the following subsections these four modules are explained in detail.

\subsection{The Solar Panel and the Mounting System}

For any solar powered lighting system, the panel size is generally determined based on the parameters such as the power rating of load that needs to be driven, the time that the load needs to be run, and the number of average sun hours per day in the respective region. This system uses a 12V 20W DC LED flood light as the load for testing which will run for 7 hours. To determine the required solar panel sizing the following assumptions and calculations were carried out.

Maximum power consumption by the load per day

$=$ Maximum power required by load $\times$ Run time of the load

$=25 \mathrm{~W} \times 7 \mathrm{Hr}(25 \mathrm{~W}$ is the maximum power required by the load $)$

$=175 \mathrm{WHrs}$

Number of hours of sunlight $=5$ Hours (Average sunlight hours in San Francisco State University)

The output power required from the solar panel is the maximum power consumption per day divided by the number of sunlight hours:

Required power output $=175 / 5=35 \mathrm{~W} / \mathrm{Hr}$

Since solar panels can be used at only $20 \%$ efficiency the resultant power loss must be considered while calculating the panel size. Hence the required final power output from the panel is:

Required power output including system losses

$=35 \times 1.2$ (power loss factor)

$=42 \mathrm{~W} / \mathrm{Hr}$

While the calculated solar panel output for this system is $42 \mathrm{~W}$, taking the power loss factor of the battery pack into consideration, a $50 \mathrm{~W} 12 \mathrm{~V}$ solar panel is chosen to power the system. This solar panel is mounted on a pole, using a pole mounting system that is easy to install and uninstall. A mounting system is used to mount the solar panel on a pole. This mounting system can withstand wind of speed up to $80 \mathrm{mph}$.

\subsection{Rechargeable Li-ion Battery Pack}

In a solar powered lighting system, the energy storage device plays a vital role in making it efficient and portable. Since the system designed is used to power DC LED lights, the energy generated by the solar panel is used only after sunset. Hence, an energy storage system is required to store the energy during the day and use it at night. Traditionally, Lead-acid batteries are used as energy storage devices for solar powered systems. These batteries are heavy and require a lot of space. They are also slow and inefficient in charging and discharging. In comparison, Li-ion batteries are more versatile. However, their battery life is shorter, 
they have extended cycle life and can be charged fast and efficiently. Li-ion batteries also have low energy wastage compared to Lead-acid batteries. Hence a custom-made Li-ion battery pack is designed and used as the energy storage device for this system.

The battery pack is custom designed using 18650 rechargeable Li-ion cells and a Battery Management System circuit (Fig. 5). Each 18650 cell has an ideal voltage of $3.7 \mathrm{~V}$ and a maximum voltage of $4.2 \mathrm{~V}$. To increase the voltage rating, multiple cells are connected in series. To increase the amperage, multiple cells are connected in parallel. Since a $12 \mathrm{~V}$ DC LED light is used as the load, an output voltage in the $12 \mathrm{~V}$ range is required. The following calculations were carried out to determine the arrangement of the cells to obtain the required voltage rating.

To get $12 \mathrm{~V}$ out of 18650 cells 3 cells need to be connected in series.

3 fully charged cells in series produce $=3 \times 4.2 \mathrm{~V}=12.6 \mathrm{~V}$ (maximum output)

Alternatively, 3 ideally charged cells produce $=3 \times 3.7=11.1 \mathrm{~V}$ (ideal output)

Each cell has an amperage of 2700 milli Amp hours. 9 rows of 3 series cells connected in parallel produce:

9 rows of 3 series cells in parallel have a capacity $=9 \times 2.7 \mathrm{Ahr}=24.3 \mathrm{Ahrs}$

The power generated from this 3series 9parallel (3S 9P) battery pack is determined by the formula:

Power $=$ Capacity $\times$ Voltage

Power generated per day $=24.3 \mathrm{AHr} \times 11.1 \mathrm{~V}=270 \mathrm{Whrs}$

Since Li-ion batteries can only be used up to $70 \%$ to $80 \%$ efficiency, the leakage power is:

Power loss (approximately 25\% of total power generated) $=267 \times 0.25=67 \mathrm{Whrs}$

Total power generated by this battery pack = power generated per day - power loss $=270-67=203 \mathrm{~W}$

Total run time of the battery pack $=$ total power generated $/$ power consumed by load $=(203) / 25=8.12$ Hours

As a result of the above calculations, a 3 series and 9 parallel combination of 18650 cells is used to power the load at full capacity for over 8 hours. Since the user has control over the power usage of the load, the 3S 9P battery pack is suitable to power the system for 7 hours. The Battery Management System (BMS) circuit attached to the battery pack ensure the cells are not overcharged or undercharged and protects the battery pack from any accidental short circuit.

The cells in the battery pack were connected using Nickle strips and resistor leads as the bus bars to protect the battery from a short circuit or overcharging.

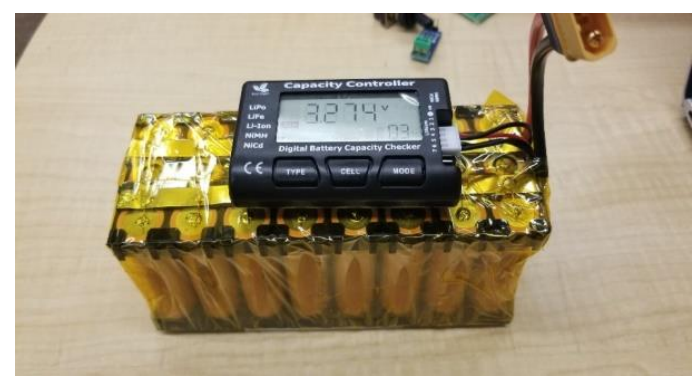

Fig. 5. The assembled 18650 battery pack with a balance charger.

\subsection{The Control Unit}

The control unit is the third module of this project. It houses all the electronic components that serve as 
the link between the solar panel, the battery, the load and the user. This unit houses the following components:

- A solar charge controller.

- PCB with the microcontroller and other components.

- The battery pack.

- The switch between manual and app mode.

- A plug to connect the load.

The Solar charge controller connects the solar panel to the battery and the battery to the load. The role of solar charge controller is to stabilize the voltage and monitor the amount of power being delivered to the battery from the solar panel. There are two types of solar charge controllers: PWM (Pulse Width Modulation) and MPPT (Maximum Power Point Tracking). PWM charge controllers charge the battery in a gradually decreasing manner as the battery approaches full charge state, essentially helping the battery get more fully charged and increasing the battery lifetime. MPPT charge controllers convert excess voltage generated by the solar panels into amperage, reducing the time required for battery to fully charge and keeping the battery voltage level optimal. MPPT charge controllers are typically more expensive compared to PWM charge controllers.

In this project, a PWM charge controller is used as it is economical and serves the purpose of charging the batteries fully and improving their life cycle. The charge controller has 6 pins two pins for the positive and negative terminals of the solar panel, two for the positive and negative terminals of the battery pack and two for the positive and negative terminals of the load, (in this case the LED light). Since the LED only needs to be turned on during the night and off in the morning, it is connected to the load terminals through a daylight sensor.

The daylight sensor has three terminals load (red), neutral (white) and line (black). The line terminal is connected to the negative load terminal on the charge controller. The neutral is connected to the positive terminal and the load is connected to the positive terminal of a 3-pin plug. The negative end of the plug is connected to the common ground on the PCB.

The complete unit is housed in an IP65 rated; weatherproof enclosure shown in Fig. 6. The enclosure can be mounted on a pole near the solar panel. The PCB circuit is shown in Fig. 7. The main component of the PCB is the NodeMCU microcontroller. A TIP122 transistor is used to switch the load on/off based on the signal received from the microcontroller. This transistor takes an input voltage of $5 \mathrm{~V}$ to switch OFF to $0 \mathrm{~N}$. The NodeMCU output voltage is only $3.3 \mathrm{~V}$, a $3.3 \mathrm{~V}$ to $5 \mathrm{~V}$ voltage level shifter is required between the microcontroller and the transistor. Pin D8 on the microcontroller is connected to the base of the transistor through a $1 \mathrm{~K} \mathrm{Ohm} \mathrm{resistor.} \mathrm{The} \mathrm{negative} \mathrm{terminal} \mathrm{of} \mathrm{the} \mathrm{load} \mathrm{in} \mathrm{this} \mathrm{case} \mathrm{the} \mathrm{negative} \mathrm{terminal} \mathrm{of} \mathrm{the} \mathrm{LED} \mathrm{is}$ connected to the collector of the transistor and the emitter is connected to a common ground from the microcontroller.

\subsection{Android and iOS Applications}

The proposed lighting system design utilizes a NodeMCU microcontroller which is based on the ESP8266 Wi-Fi module. The NodeMCU is used to connect the system to the internet, enabling the user to control and monitor it remotely. A cross platform Android/iOS application is developed for remote control and surveillance. To access a system through the mobile application user authentication and secure sign in are required. The Android app and the iOS app are connected to the Google Firebase cloud console for data handling, storage and motoring. The data collected by the system is pushed to the Google cloud over the Internet. The applications are designed to control various aspects of the LED light with two operating modes: Power saver and Manual. In power saver mode the LED is switched on at $30 \%$ brightness using 
PWM signal from the microcontroller. In Manual mode the LED can be turned on at any level of brightness. The user can adjust the brightness using the brightness control bar in the mobile application.

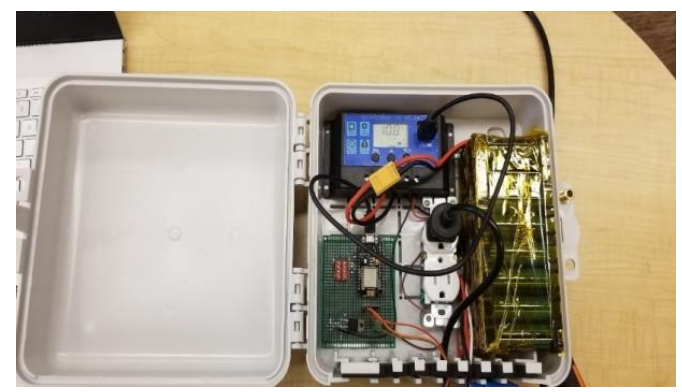

Fig. 6. The control hub with the PCB, the battery and the charge controller

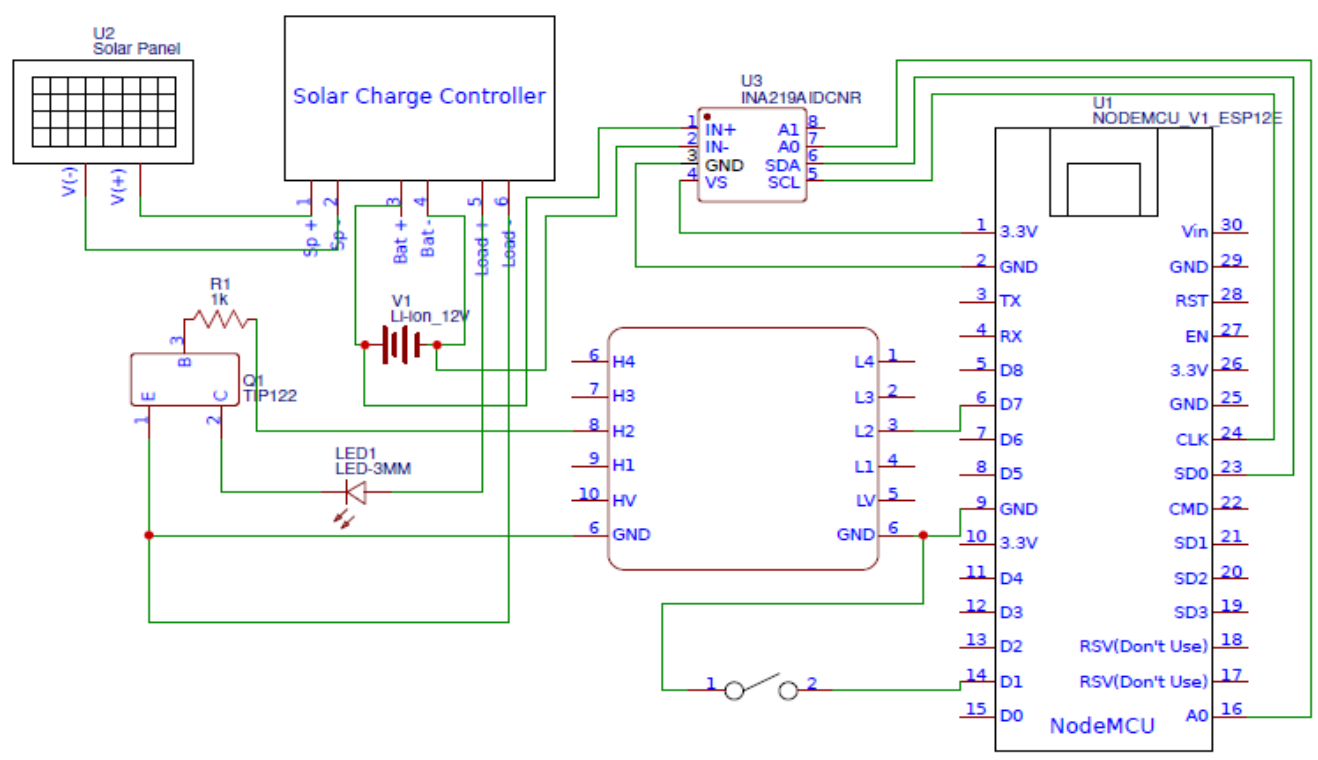

Fig. 7. The PCB design of the control circuit.

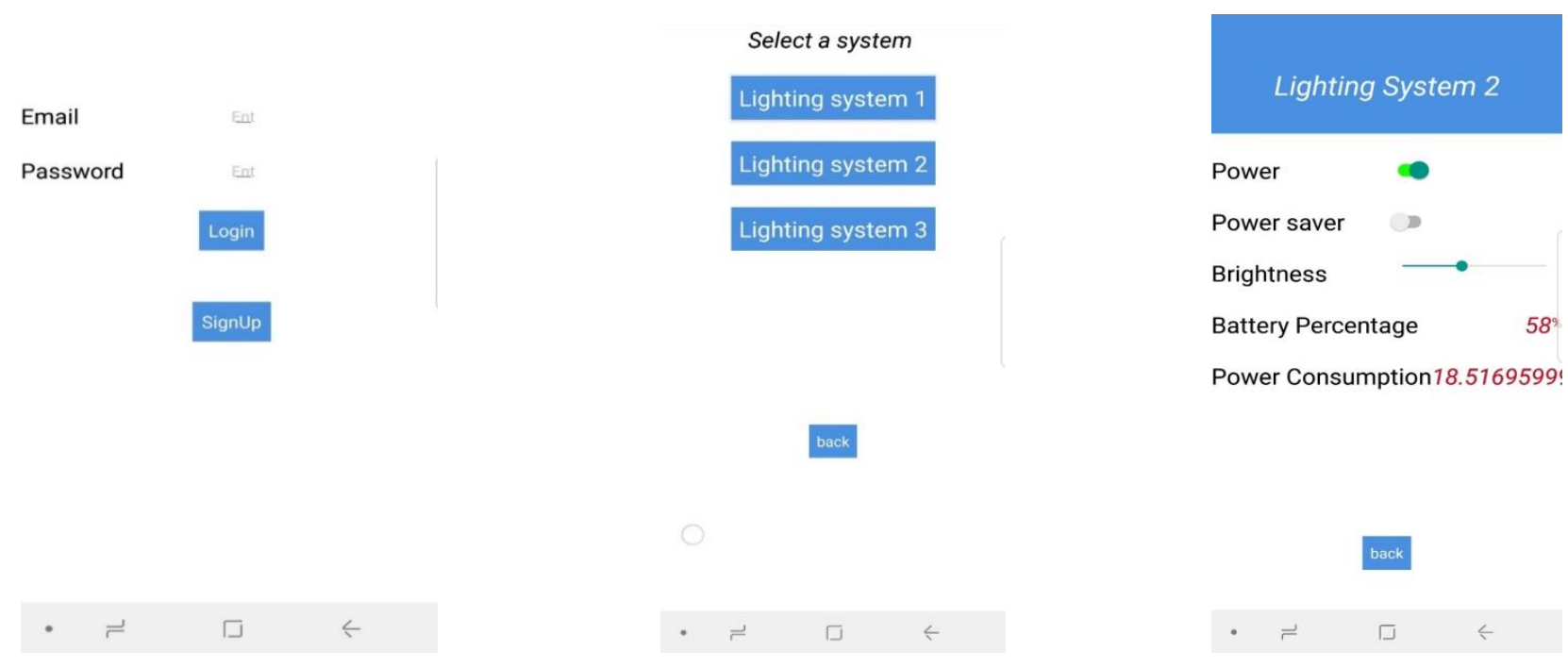

Fig. 8. The Login screen of the mobile application.
Fig. 9. The screen to choose a lighting system.
Fig. 10. System control panel. 
To connect the system to multiple Wi-Fi networks the WiFiManager library is used. This enables the system to connect to different Wi-Fi networks on the go without having to change the credentials in the software itself. The mobile application has four screens: the login screen, the signup screen, the system selection screen and the control panel for each one of the systems shown in Fig. 8, 9 and 10.

\section{Assembly and Testing}

The lighting system design discussed is implemented in two lighting systems. Both systems powered 12V 20W DC LED floodlights. Both of the systems were tested over a period of 4 months. They were tested for the number of hours the load could run, the power generated by the solar panels in different weather conditions, the reliability of the wireless control and the working of the android/iOS applications. During these 4 months the data collected from the sensors on power generation and consumption is stored on the cloud in the Google Firebase database.

\section{Results}

The two systems have been successfully tested for the ease of control, reliability and accessibility. The power consumption data is also monitored and logged on a day to day basis in the Google Firebase database. The data is collected in half hour intervals to reduce the power consumption of the microcontroller, this collected data is represented graphically as shown in Fig. 11 and Fig. 12.

From the graphs it can be observed that on cloudy and rainy days the power generated was substantially less compared to sunny days.

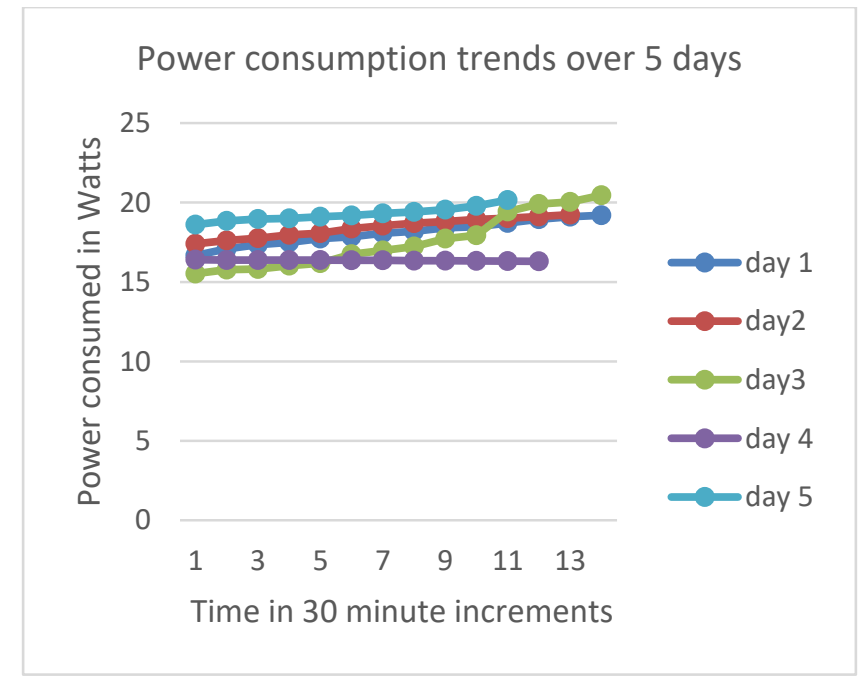

Fig . 11. The graph representing power consumption data over a period of 5 days.

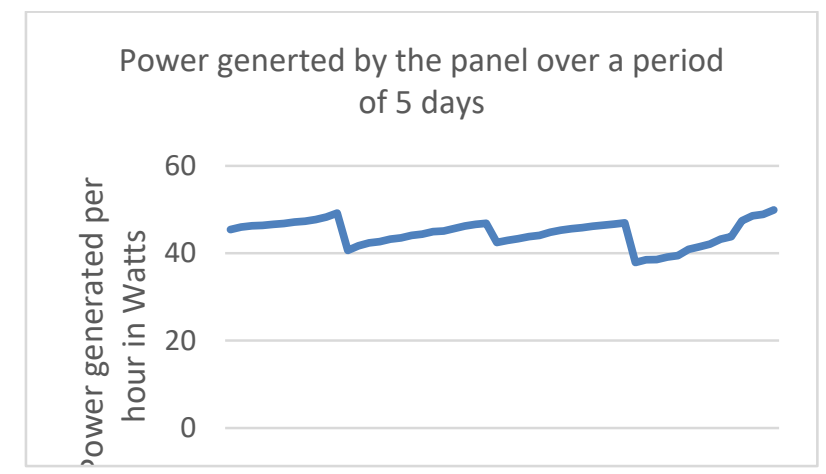

Fig. 12. The power generated per hour by the solar panel over a period of 5 days. 
The mobile application works seamlessly without any loss of data as long as the system is connected to the internet. By using a single control hub to power one or more LEDs the cost of producing such lighting systems is substantially reduced. The cost of making one such system including the solar panel and other peripherals is approximately $\$ 650$ which is much cheaper when compared to the available options in the market. The use of Google Firebase as the backend enabled an economical and secure means of communicating and storing the information.

\section{Conclusion}

The lighting system design can be used to power multiple lights from a single control hub. Its modular design allows for easy expansion of the system with minimal effort. Use of a single system which can power multiple LEDs is a more economical way of implementing solar powered lighting in smart cities, campuses and parks. Further, use of Li-ion batteries makes the system more robust and requires a much smaller number of maintenance and service runs. The design of the backend of the system facilitates the use of the system as a test bench to collect valuable data on the amount of power generated, and power consumed. This data can be used to better manage the system and save resources and energy.

For future work, the system's functionality can be expanded by adding peripherals like a security camera, PIR and air quality sensors. The security camera feed and the sensor readings can be made available to the user directly in the mobile application. Also, this data can be stored in the cloud database. As videos occupy more space than any other data, implementing ring buffer technique helps save space. i.e. writing data in memory and overwriting that data after a fixed period of time. Further, low power consuming wireless communication technologies like LoRa WAN and Bluetooth Low Energy (BLE) can be utilized to develop the control network of street lights.

\section{Conflict of Interest}

The authors declare no conflict of interest.

\section{Author Contributions}

Sairoop Dasaraju is a master's graduate student at San Francisco State University carrying out this research project with his supervisor professor Hamid Shahnasser.

\section{References}

[1] International Energy Association. (2018). Global energy demand grew by 2.1\% in 2017, and carbon emissions rose for the first time since 2014. Retrieved from https://www.iea.org/news/global-energy-demand-grew-by-21-in-2017-and-carbon-emissions-rose-fo r-the-first-time-since-2014

[2] Northeast Group, llc. (2016, February 25). Global LED and smart street lighting: Market forecast (2017-2027). Retrieved from http://www.northeast-group.com/reports/Brochure-Global\%20LED\%20and\%20Smart\%20Street\%2 0Lighting-Market\%20Forecast\%202017-2027\%20-\%20Northeast\%20Group.pdf

[3] Gupta, V., Thakur, K., \& Thakur, R. (2015). Based smart street lights. International Journal of Research (IJR), 2(10).

[4] Long, X., \& Jing, Z. (2008). An intelligent driver for light emitting diode street lighting. Proceedings of the 2008 World Automation Congress.

[5] Chen, P.-Y., et al. (2008). Development of an energy efficient street light driving system. Proceedings of the 2008 IEEE International Conference on Sustainable Energy Technologies. 
[6] Li, F., et al. (2009). LEDs: A promising energy-saving light source for road lighting. Proceedings of the 2009 Asia-Pacific Power and Energy Engineering Conference.

Copyright (C) 2020 by the authors. This is an open access article distributed under the Creative Commons Attribution License which permits unrestricted use, distribution, and reproduction in any medium, provided the original work is properly cited ( $\underline{\text { CC BY 4.0) }}$.

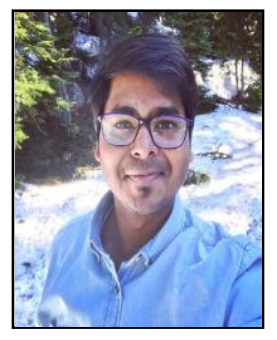

Sairoop Dasaraju was born on November 05, 1993. He obtained his master's degree in embedded electrical and computer systems engineering from San Francisco State University, San Francisco, CA, USA; and the master's in radar and microwave engineering from Andhra University, Visakhapatnam, AP, India in May 2016. His bachelor's degree in electronics and communication engineering was also awarded by Andhra University, Visakhapatnam, AP, India in May 2014.

Mr. Sairoop is now a graduate teaching assistant at San Francisco State University.

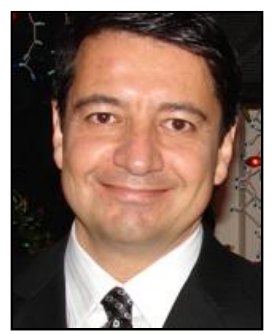

Hamid Shahnasser is hold with the B.E. degree in electrical engineering from McGill University, Montreal; the MS degree in electrical and computer engineering from Carnegie-Mellon University; and the Ph.D. from Drexel University Pennsylvania.

Dr. Shahnasser is currently a professor of electrical and computer engineering at San Francisco State University, and the School of Engineering graduate program coordinator. His areas of interest are IOT, communication networks and computer systems.

Dr. Shahnasser has been a research faculty consultant to NASA Ames Research Center projects since 1990, where he collaborated on several research grants projects. Professor Shahnasser has also been recipient of grants from NSA, Department of Education, National Science Foundation and various private companies carrying out research in his areas of interest. 